\title{
Optimal correction of data from misoriented multi-component geophysical sensors
}

\author{
Lars KRIEGER $^{1}$, Francesco GRIGOLI ${ }^{2}$ \\ ${ }^{1}$ Department of Earth Sciences, University of Adelaide, Adelaide, Australia \\ ${ }^{2}$ Institute of Earth and Environmental Sciences, University of Potsdam, Potsdam, Germany
}

\begin{abstract}
Incorrectly oriented geophysical sensors affect data analysis procedures, which can lead to errors in results and interpretations. These problems generally occur in applications, in which the orientation of the sensor cannot be actively controlled and is not known a priori. Common examples are sensors deployed in borehole installations or on the seafloor. We introduce two methods to optimally correct data sets from misaligned two- and three-component sensors. Firstly, we demonstrate how a set of multiple twocomponent sensors can be re-oriented in a single step calculation. In the second part, we introduce a quaternion-based analytical method for the calculation of the optimal re-orientation of three-component sensors. A common approach to the re-orientation of three-component data is to assume that the vertical axis does not have to be corrected during the processing. We show, that this approximation can lead to significant deviations between re-oriented data and original data. Therefore, the estimation of not only an optimal rotation angle, but also the rotation axis can help to mitigate a systematic source of error in the processing of geophysical data.
\end{abstract}

\section{INTRODUCTION}

For a start, we consider two-component timeseries data from geophysical sensors of a priori unknown orientation, and we are interested in the re-orientation of these sensors with respect to a given reference. The data commonly result from the assumption that the vertical axis of a threecomponent sensor is correctly determined, and therefore the signal from that respective component can be excluded from the correction process. This is a common approach and it is used in many applications. We show that the optimal misalignment-correction for such a two-component data set can be estimated in a single, non-iterative calculation step ${ }^{1)}$. The result is optimal with respect to the $1_{2}$ norm of the overall deviation between the re-oriented and the optimal data.

Although the simplification of neglecting the third sensor component is commonly applied, and the resulting deviations are assumed to be negligible, it is still beneficial to take the full information contained in the data sets into account. Therefore, we have developed a reorientation algorithm for multi-component geophysical sensors (i.e. two- and three-component sensors). In the second part of this presentation, we introduce this quaternion-based method, which yields an analytical solution for the (relative) reorientation of sensors in three dimensions. We show that our method is not only fully functional, but also that it is superior to the commonly applied approach of a grid search over the parameter space for finding optimal rotation parameters ${ }^{2}$.

We also demonstrate that the assumption a potential deviation of the vertical sensor component from the true vertical only leads to small errors in the result is not necessarily true, so it is important to rely on an analytical estimate of reorientation parameters and to include all sensor components in the process. By introducing our new approach to the correction of sensor mis-alignment, we can overcome the dependency on approximative solutions and eliminate systematic sources of errors in standard time-series data processing.

\section{TWO-COMPONENT DATA: SINGLE STEP RE-ORIENTATION}

We have a set of seismic sensors, and we assume that their vertical components are parallel. For example they are parallel to the casing of the vertical borehole in the case of VSP data, or perfectly coupled with the seafloor and leveled for OBS data. Only the orientation of the two horizontal orthogonal components is a priori unknown. Then the alignment of the sensors requires a rotation of the horizontal components (X and $\mathrm{Y}$ ) around the vertical axis. Our aim is to determine the relative orientation of a particular subset of seismic sensors with respect to a chosen reference sensor. 


\section{(1) Complex traces}

The two components $\mathrm{X}$ and $\mathrm{Y}$ are orthogonal, the recorded values are real, and the $\mathbb{R}^{2}$ and $\mathbb{C}$ are isomorph. Hence, the two-component real valued signal can be interpreted as a complex valued signal $\mathrm{S}(\mathrm{t})$ instead:

$$
S(t)=X(t)+i \cdot Y(t)=A(t) \cdot e^{i \varphi(t)}
$$

with $A(t)=|S(t)|$ and $\varphi(t)=\arg (S)$. The signal $\mathrm{S}(\mathrm{t})$ is then called complex trace. Another point is that a digitally recorded seismic trace is a discrete time-series with $\mathrm{N}$ samples, therefore we can write $\mathrm{S}(\mathrm{t})$ as an $\mathrm{N}$-vector:

$$
\begin{aligned}
\mathbf{S} & =\mathbf{X}+i \cdot \mathbf{Y} \\
S_{j} & =X\left(t_{j}\right)+i \cdot Y\left(t_{j}\right), \quad S_{j} \in \mathbb{C}, j=1 \ldots N
\end{aligned}
$$

\section{(2) Sensor re-orientation}

Due to the isomorphism $\mathbb{R}^{2} \sim \mathbb{C}$, a vector $\mathrm{v} \in \mathbb{R}^{2}$ can be equivalently described by a complex number $\mathrm{z} \in \mathbb{C}, \mathbf{v} \simeq z$. A clockwise rotation of $\mathrm{v}$ by an angle $\psi$ is therefore equivalent to a complex multiplication:

$\mathbf{v} \stackrel{R o t}{\mapsto} \mathbf{v}^{\prime} \Leftrightarrow z \mapsto z \cdot e^{i \psi}$

A rotation of the signal from a two-component sensor ( eq. 1) can therefore be expressed as

$$
\begin{aligned}
S(t) \mapsto S^{\prime}(t) & =S(t) \cdot e^{i \psi} \\
& =A(t) \cdot e^{i(\varphi(t)+\psi)} .
\end{aligned}
$$

As before, this relation can be expressed in form of an $\mathrm{N}$-vector (eq. 2) for each sensor $\mathrm{k}$ :

$$
\left(\begin{array}{c}
{ }^{k} S_{1}^{\prime} \\
{ }^{k} S_{1}^{\prime} \\
\vdots \\
{ }^{k} S_{N}^{\prime}
\end{array}\right)=\left(\begin{array}{c}
A_{1}^{k} e^{i\left(\varphi_{1}^{k}+\psi\right)} \\
A_{2}^{k} e^{i\left(\varphi_{2}^{k}+\psi\right)} \\
\vdots \\
A_{N}^{k} e^{i\left(\varphi_{N}^{k}+\psi\right)}
\end{array}\right)
$$

We take a set of $\mathrm{K}$ sensors, and $\mathrm{J}=\mathrm{K}-1$ angles $\psi_{j}(j=1 \ldots J)$ between the X-components of the respective sensors. To align the $\mathrm{j}$-th and $(\mathrm{j}+1)$-th sensor, we have to rotate the latter by $-\psi_{j}$, equivalent to the multiplication of the complex trace by $e^{i \psi_{j}}$. Therefore, we face the inverse problem of inferring the optimal value for $e^{i \psi_{j}}$ from the minimisation of the misfit between the complex traces of the $j$-th and $(j+1)$-th sensor.

Since we are interested in the overall re-orientation of all sensors with respect to one reference sensor, we extend this concept. The stations are arbitrarily enumerated, and then we consider the rotation angles between consecutive station pairs.
Now, we define a data vector $\mathbf{d}$ that contains all discrete complex traces from the first K-1 stations

$\mathbf{d}=\left({ }^{1} \hat{S}_{1},{ }^{1} \hat{S}_{2}, \ldots,{ }^{1} \hat{S}_{N},{ }^{2} \hat{S}_{1}, \ldots,{ }^{K-1} \hat{S}_{N}\right)^{T}$

and a sparse data matrix $\mathbf{G}$ containing discrete complex traces from the last $\mathrm{K}-1$ stations

$$
\mathbf{G}=\left(\begin{array}{cccc}
{ }^{2} \hat{S}_{1} & 0 & \ldots & 0 \\
{ }^{2} \hat{S}_{2} & 0 & \ldots & 0 \\
\vdots & \vdots & \ddots & \vdots \\
{ }^{2} \hat{S}_{N} & 0 & \ldots & 0 \\
0 & { }^{3} \hat{S}_{1} & \ldots & 0 \\
0 & { }^{3} \hat{S}_{2} & \ldots & 0 \\
\vdots & \vdots & \ddots & \vdots \\
0 & 0 & \ldots & { }^{K} \hat{S}_{1} \\
\vdots & \vdots & \ddots & \vdots \\
0 & 0 & \ldots & { }^{K} \hat{S}_{N}
\end{array}\right) .
$$

We assume that the main signal wavelengths are significantly larger than the inter-station distances, so that the signals from different stations are identical, and only their components are rotated with respect to each other. Therefore, the data vector $\mathbf{d}$ and the data matrix $\mathbf{G}$ are related by the respective rotations, expressed as multiplications by normalised complex numbers:

$\mathbf{d}=\mathbf{G} \cdot \mathbf{m}, \quad \mathbf{m}=\left(e^{i \psi_{1}}, \ldots, e^{i \psi_{k-1}}\right)^{T}$.

For real data, re-orientation of the sensors is therefore equivalent to solve a system of linear equations for the rotation parameter vector $\boldsymbol{m}$ :

$\mathbf{d}=\mathbf{G} \cdot \mathbf{m}+\mathbf{r}$,

with an additional data error vector $\boldsymbol{r}$.

$\mathbf{G}$ is a complex valued and non-square matrix, so the inverse problem is generally overdetermined, and can be directly solved by calculating the generalized inverse of G. For complex valued matrices the generalized inverse can be obtained using the hermitian transpose $\mathbf{G}^{H}$. If $\mathbf{G}^{H} \mathbf{G}$ is nonsingular, the optimal estimator for the vector of pairwise rotation parameters $\mathbf{m}_{l_{2}}$ is obtained by

$\mathbf{m}_{l_{2}}=\left(\mathbf{G}^{H} \mathbf{G}^{-1}\right) \cdot \mathbf{G}^{H} \mathbf{d}$

After the determination of $\mathbf{m}_{l_{2}}$, the rotation angles are known as well: $\psi_{n}=\arg \left(\mathbf{m}_{l_{2}, n}\right)$.

By simply concatenating all discrete data traces from all sensors, we have estimated the pairwise inter-station rotation angles for the whole set of sensors in a single step calculation. The re- 
orientation angles for each individual station with respect to a chosen reference are obtained by successively adding the resulting relative rotation angles.

\section{(3) Example}

The Graefenberg Array is a permanent seismological array located in the south east of Germany, it consists of 13 three-component stations. To check the correct orientation of the stations forming the array, we used a teleseimic reference event (Aleutian Islands, $\mathrm{M}_{w}=7.4,24 / 06 / 2011$; data are frequency low-pass filtered at $0.03 \mathrm{~Hz}$ ). We compared data from different array stations and found that station GRA1 yielded inconsistent signals compared to other stations (c.f. Fig.1).

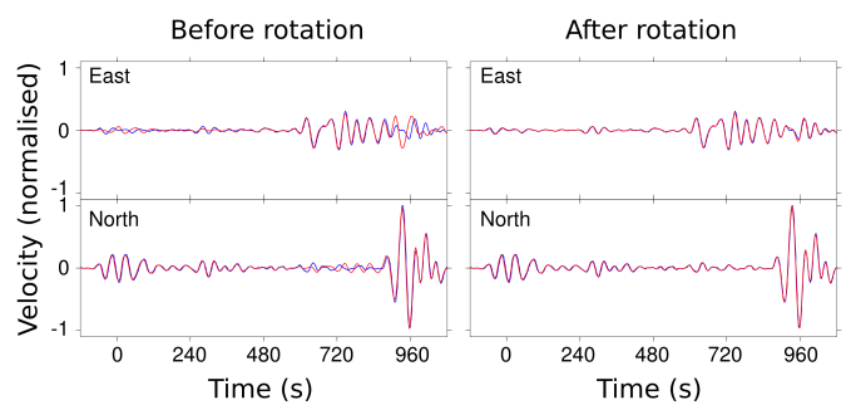

Figure 1 Comparison of velocity seismograms for the teleseismic reference event (horizontal components), GRA1 (red) vs. GRA3 (blue). Left: original signals. Right: re-oriented sensor data (data from corrected GRA1 is consistent with GRA).

The signal differences between stations as well as the effect of sensor re-orientation becomes more apparent in a hodogram representation of the complex traces as visualised in Fig. 2.

By applying our method on the array data of the reference event, we found that most inter-station mis-orientations were negligible within the estimated data error range. However, rotation angle estimates between station GRA1 and other stations showed a significant deviation. We found a rotation angle of $\sim 18^{\circ}$, and a correction of data from GRA1 using our estimated angle indeed yielded a signal that is consistent with data from other stations, see Figs. 1 and 2.

Our findings can be explained by the maintenance history of the problematic sensor. The Station GRA1 was updated on September 2011, and during the updating works the orientation mark of the station was lost. For this reason it was re-installed with an erroneous orientation. We applied our methodology to a teleseismic event that had occurred before 2011, and we found that station GRA1 was correctly oriented before that date.

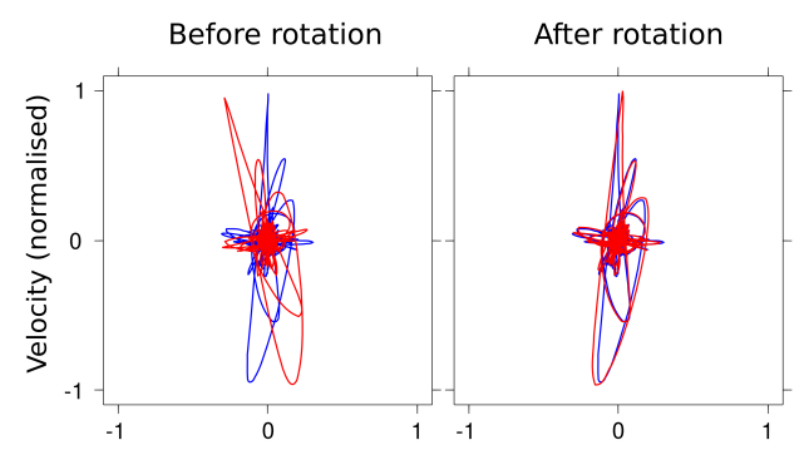

Figure 2 Normalised hodogram representation of the velocity seismogram comparison in Fig. 1.

\section{THREE-COMPONENT DATA: OPTIMAL SENSOR RE-ORIENTATION}

It is a common assumption that the vertical component of a geophysical sensor is sufficiently well oriented, but this is not easily verifiable. Thus, it remains unclear if this uncertainty really is negligible for all cases. Therefore, a complete sensor re-orientation consists not only of finding the optimal rotation angle but also the respective rotation axis. However, not many approaches include the third (vertical) component in the reorientation process during standard data processing and analysis ${ }^{2}$.

We overcome principle disadvantages of the existing (numerical) solution schemes for the rotational correction of three-component time-series data by expressing using quaternions. This allows us to calculate an analytical solution for an optimal geophysical sensor re-orientation in three dimensions. Unlike other approaches, this method utilises the full waveform data of the signal timeseries, therefore it is not restricted to the analysis of seismic traces, but can be applied to all two- or three-component time-series data. Additionally, it allows us to calculate uncertainties for the estimated re-orientation parameters.

\section{(1) Quaternions}

Quaternions are a set of numbers $\mathbb{H}$ introduced by Hamilton 1853. They form a division algebra over $\mathbb{R}^{4}$ and due to the isomorphism $\mathbb{H} \simeq \mathbb{R}^{4}$, elements of the quaternion field $\mathbb{H}$ may be interpreted as elements of a four dimensional real vector space, which is a higher dimensional equivalent to the relation between $\mathbb{R}^{2}$ and $\mathbb{C}$. Like complex numbers, elements $\mathbf{q} \in \mathbb{H}$ can be written as a combination of a scalar (real) and a vector (imaginary) part:

$$
\underset{\sim}{\mathbf{q}}=\left(q_{0},\left[q_{1}, q_{2}, q_{3}\right]\right)=:\left(s_{\mathbf{q}}, \mathbf{v}_{\mathbf{q}}\right) \in \mathbb{R} \times \mathbb{R}^{3} .
$$


We can identify the $\mathbb{R}^{3}$ with the subspace ${ }^{i} \mathbb{H} \subset \mathbb{H}$ of vanishing real parts so each three-dimensional vector is equivalent to one quaternion. The quaternion addition and scalar multiplication are defined component-wise. Additionally, there is a pair-wise quaternion product $\otimes$ connecting two quaternions $\underset{\sim}{\mathbf{q}, \mathbf{p}}$ :

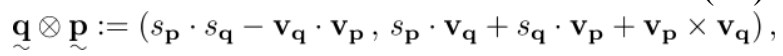

with the inner product ".", as well as the standard outer three dimensional vector cross product " $x$ ".

Consider a rotation that transforms $\mathbf{x}$ into $\mathrm{x}^{\prime}$ $\left(\mathbf{x}, \mathbf{x}^{\prime} \in \mathbb{R}^{3}\right)$. This operation is defined by the rotation angle $\alpha \in\left[0,360\left[\right.\right.$ and a unit vector $\hat{\boldsymbol{\rho}} \in \mathbb{R}^{3}$ in direction of the rotation axis. By interpreting the three-dimensional vectors $\mathbf{x}$ as elements $\underset{\sim}{\mathbf{x}} \in{ }^{i} \mathbb{H}$, we can express the rotation in terms of quaternions: We know $^{3)}$ that there is a unit quaternion $\hat{\mathbf{q}}$ with an involution $\overline{\hat{\hat{q}}}$ so that

$$
\underline{x}^{\prime}=\underset{\sim}{\hat{\mathbf{q}}} \otimes \underset{\sim}{\mathbf{x}} \otimes \underset{\sim}{\overline{\hat{\mathbf{q}}}} .
$$

The quaternion, which describes a rotation of an angle $\alpha$ about an axis $\hat{\boldsymbol{\rho}}$ is given by the normalisation of

$$
\underset{\sim}{\mathbf{q}}=\left(\cos \frac{\alpha}{2}, \sin \frac{\alpha}{2} \cdot \hat{\boldsymbol{\rho}}\right) .
$$

\section{(2) Optimal re-orientation}

We consider orthogonal three-component timeseries data sets, native two-component data have to be extended by adding a third zero-valued component. The data are finitely sampled and of finite length $\mathrm{N}$. If $\mathbf{p}(\mathrm{t})$ are the original and $\mathbf{r}(\mathrm{t})$ the rotated data, we have two vectors $\left(\mathrm{p}_{1}, p_{2}, p_{3}\right),\left(r_{1}, r_{2}, r_{3}\right) \in \mathbb{R}^{3}$ for each point in time $\mathrm{t}_{n}$. The time series are therefore equivalent to sets of points $\left\{\mathbf{p}_{n}\right\},\left\{\mathbf{r}_{n}\right\}(n=1 \ldots N)$. Since we are only correcting for rotation between sensors without additional offsets, all data must be corrected for a mean value of zero.

For a normalised comparison of the deviation between two data sets $\mathbf{d}_{\mathbf{1}}, \mathbf{d}_{\mathbf{2}}$ we express the overall deviation as the percentage residual "Res":

$$
\text { Res }:=\frac{\left\|\Delta_{\mathbf{d}_{1}, \mathbf{d}_{2}}\right\|}{\left\|\mathbf{d}_{\mathbf{1}}\right\|} \cdot 100 \text {. }
$$

with $\Delta_{\mathbf{d}_{1}, \mathbf{d}_{2}}:=\mathbf{d}_{\mathbf{2}}-\mathbf{d}_{1}$. Now we find a rotation operator, which minimises the deviation from $\left\{\mathbf{r}_{t}\right\}$ when applied to the set $\left\{\mathbf{p}_{t}\right\}$. The solution can be inferred from the original data in a few simple calculation steps (the derivation can be found in publications on the theory of quaternions):

1. Sum over outer products of both data sets for all samples

$$
\mathbf{S}:=\sum_{l=1}^{L}{ }^{l} \mathbf{p} \cdot{ }^{l} \mathbf{r}^{T} .
$$

2. Re-arrange the contents of $\mathbf{S}$ into a $4 \times 4$ matrix $\mathbf{N}$

3. Calculate the eigenvalues and -vectors of $\mathbf{N}$.

4. The rotation quaternion is the normalised eigenvector of the largest eigenvalue:

$$
\stackrel{\hat{\mathbf{q}}_{\text {opt }}}{\sim}=\frac{\stackrel{\nu_{1}}{\sim}}{\left\|\boldsymbol{\nu}_{\mathbf{1}}\right\|}, \quad \mathbf{N} \cdot \boldsymbol{\nu}_{\boldsymbol{i}}=\lambda_{i} \cdot \boldsymbol{\nu}_{\boldsymbol{i}}, i \in\{1, \ldots, 4\}
$$

5. Sensor re-orientation can be directly calculated by a quaternion multiplication.

6. Rotation axis and angle can be obtained from the unit quaternion $\hat{\nu}_{1}$.

\section{(3) Example}

We demonstrate our method using synthetic seismic data for a vertical seismic profile (VSP) geometry. Within the VSP, six sensors are vertically aligned, but the relative orientation of the sensors is unknown. We use the $\mathrm{E} \mathrm{D}^{4)}$ code to calculate seismograms for an arbitrarily chosen source mechanism (explosion); for the comparison of results for different signal frequency contents, we generate an additional data set by applying ${ }^{5)}$ a zerophase Butterworth low-pass frequency filter with a corner frequency of $0.1 \mathrm{~Hz}$. The geometry is shown in Fig. 3.

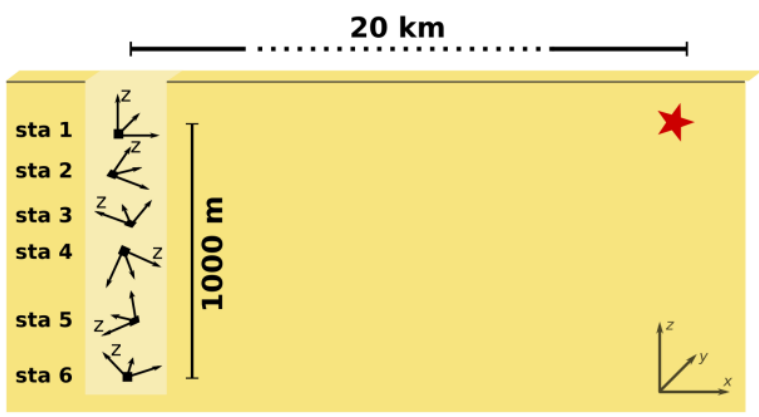

Figure 3 Vertical seismic profile geometry for the generation of synthetic seismograms. The (explosive) source location is marked by a star. Orientations of sensors 2-6 are randomised.

The data of stations 2-6 are rotated with randomly chosen angles and axes, and we carry out the reorientation of the stations 2-6 with respect to the topmost station 1. Examples of resulting re-oriented data (original and low-pass frequency filtered) are shown in Fig. 4. 

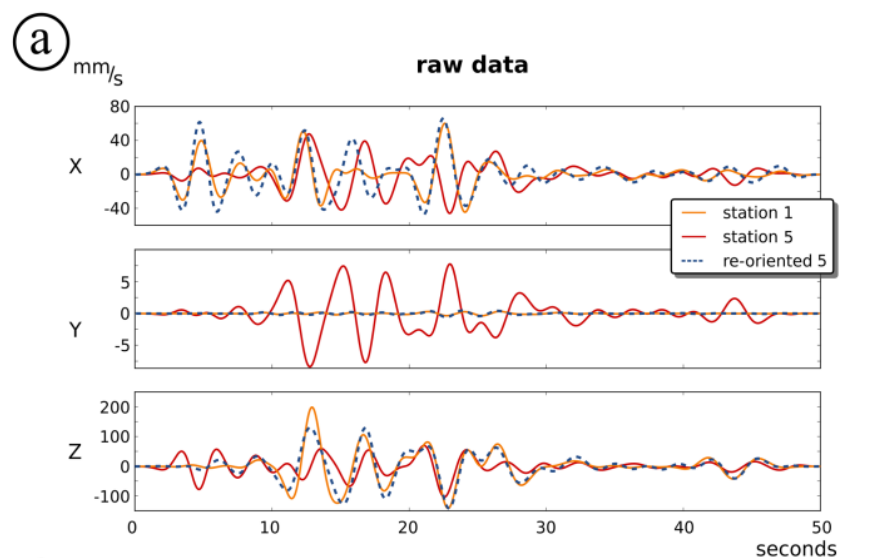

(b)

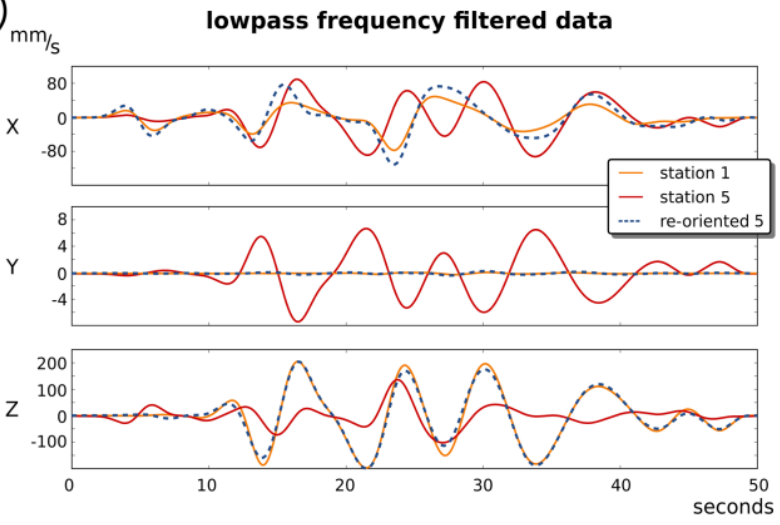

Figure 4 Example of rotated synthetic data from stations 1 and 6 in the VSP geometry (Fig. 3). a) Original data. b) Low-pass frequency filtered data. The orientation of station 5 (red) is rotated by $-93^{\circ}$ about the axis $(-48.4,-68.5,54.5)$ with respect to station 1 (orange). The result from the optimal re-orientation is shown in blue (dotted line). The estimation deviates from the correct rotation parameters for both data sets, because the original signals arriving at the two sensors are slightly different. Raw data: $1.5^{\circ}$ off in the orientation of the axis and $0.4^{\circ}$ in the rotation angle; filtered data: $1.8^{\circ}$ off in the orientation of the axis and $1.5^{\circ}$ in the rotation angle. (Figure under SEG copyright ${ }^{2}$ )

\section{(4) Comparison with a conservative grid search approach}

The standard approach for the re-orientation of three-component sensor time-series data is the reduction to two dimensions: If we assume that the vertical sensor axis is sufficiently close to the true vertical, then we can determine the optimal rotation angle around this fixed axis using various techniques.

We use a setup with six seismometers situated at the surface of a flat topography, resembling a realistic ocean-bottom-seismometer layout (see Fig. 5 ), in order to compare the quaternion method with this standard approach. We use E3D ${ }^{4)}$ to calculate a set of synthetic seismograms, and we generate an additional data set by adding white noise to the original data.

All sensors are rotated about a randomly determined axis that deviates less than $20 \%$ from the vertical, justifying the assumption of a vertical axis. The quaternion approach estimates a solution in the full three-dimensional space, so we have to set the vertical component of the data to zero for all stations in order to compare it with a grid-search for a single rotation angle. We calculate the reorientation and compare our results with the values obtained from a grid-search over $360^{\circ}$ in steps of $1^{\circ}$.

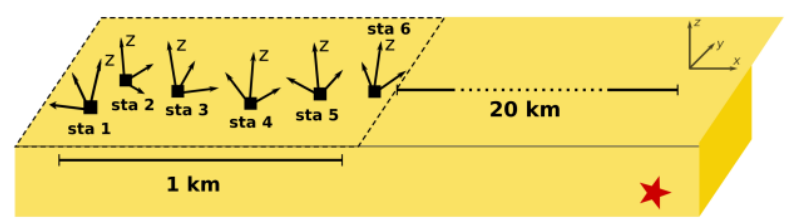

Figure 5 OBS (2D) station geometry for the generation of synthetic seismograms. The subsurface (explosive) source location is marked by a star.

The whole process is repeated many times for statistical evaluation, numerical results for an arbitrary realisation are given in Table 1 . The following general characteristics can be observed:

- The quaternion method results are better than grid search results $\left(\operatorname{Res}_{\text {Quaternion }} \leq \operatorname{Res}_{\text {Gridsearch }}\right)$.

- The differences between the quaternion method and the grid-search approach are small.

Table 1 Resulting numerical parameters for the re-orientation of data assuming a rotation about the vertical axis: comparison of the quaternion method with a grid-search (data noise levels $0 \%$ and $10 \%$ ). $\angle \hat{\boldsymbol{\rho}}$ denotes the deviation from the axis $\hat{\boldsymbol{\rho}}$. Angles tilt and $\alpha$ are given in degrees. $\Delta t$ is the time shift applied to the data before re-orientation.

\begin{tabular}{|c|c|c|c|c|c|c|c|c|c|c|c|c|}
\hline \multirow[b]{3}{*}{ Station } & \multirow{2}{*}{\multicolumn{3}{|c|}{ Original data }} & \multirow[b]{3}{*}{$\Delta t$} & \multicolumn{4}{|c|}{ Quaternion method } & \multicolumn{4}{|c|}{ Grid-search } \\
\hline & & & & & \multicolumn{2}{|c|}{ no noise } & \multicolumn{2}{|c|}{$10 \%$ noise } & \multicolumn{2}{|c|}{ no noise } & \multicolumn{2}{|c|}{$10 \%$ noise } \\
\hline & $\hat{\rho}$ & tilt & $\alpha$ & & $\alpha$ & Res & $\alpha$ & Res & $\alpha$ & Res & $\alpha$ & Res \\
\hline sta 1 & - & - & - & - & - & - & - & - & - & - & - & - \\
\hline sta 2 & $(-5.4,-5.4,99.7)$ & 4.4 & -122 & $0.08 \mathrm{~s}$ & -123.9 & 14.9 & -125.1 & 51.9 & -123.0 & 14.9 & -126.0 & 51.9 \\
\hline sta 3 & $(-23.2,-7.8,97.0)$ & 14.2 & -14 & $0.22 \mathrm{~s}$ & -10.7 & 14.3 & -12.1 & 53.5 & -11.0 & 14.3 & -12.0 & 53.5 \\
\hline sta 4 & $(3.2,12.1,99.2)$ & 7.2 & -122 & $0.42 \mathrm{~s}$ & -116.3 & 37.2 & -117.3 & 64.0 & -117.0 & 37.2 & -117.0 & 64.0 \\
\hline sta 5 & $(2.9,-8.5,99.6)$ & 5.2 & 173 & $0.70 \mathrm{~s}$ & 167.5 & 42.6 & 165.9 & 71.7 & 168.0 & 42.6 & 166.0 & 71.7 \\
\hline sta 6 & $(-15.2,-18.0,97.2)$ & 13.6 & 111 & $1.06 \mathrm{~s}$ & 96.3 & 49.0 & 95.6 & 78.8 & 97.0 & 49.0 & 96.0 & 78.8 \\
\hline
\end{tabular}


- The deviation of the re-oriented sensor data from true data can be large, even for only slightly tilted rotation axes.

- Even for small tilt angles of rotation axes the full three-dimensional re-orientation might be required.

\section{CONCLUSIONS}

We have introduced two methods for improving the re-orientation of two- and three-component geophysical sensors. We have presented optimal analytical solutions for the estimation of rotation angles between sensors under the commonly applied assumption that the main signal wavelengths are significantly larger than the interstation distances. For three-component sensors, the quaternion-based re-orientation method estimates the best rotation axis and angle. For two-component sensors, the quaternion method yields the optimal rotation angle, which is consistent with the results of a standard grid search approach. Rotated data can deviate significantly from the original data, even for small deviations of the rotation axis from the true vertical; hence the full three dimensional reorientation method is an improvement over the common approximating approaches.

Our methods use the full time-series data and do not require prior identification of specific signal characteristics, e.g. seismic phases. The methods are not restricted to seismic data, but can be applied to general time series data from sensors with orthogonal components.

ACKNOWLEDGMENT: We thank Sebastian Heimann from the Geoforschungszentrum Potsdam (GFZ) for the testing of the numerical codes. Furthermore, we deeply appreciate the advice of Prof. Anthony Roberts from the School of Mathematics at the University of Adelaide. We wish to thank Klaus Stammler and Thomas Plenefisch from BGR Hannover for useful discussions.

Parts of this work have been published in Geophysics and are under SEG copyright ${ }^{2}$.

This work has been partially realised within the research project MINE. The project MINE is part of the research and development program GEOTECHNOLOGIEN and is funded by the German Ministry of Education and Research (BMBF) - grant no. BMBF03G0737A.

\section{REFERENCES}

1) Grigoli, F., Cesca, S., Dahm, T. and Krieger, L., 2012, A complex linear least-squares method to derive relative and absolute orientations of seismic sensors, Geophysical Journal International, 188(3), 1243-1254.

2) Krieger, L., and Grigoli, F., 2015, Optimal reorientation of geophysical sensors: a quaternion-based analytical solution: Geophysics, 80(2), F19-F30.

3) Horn, B., 1987, Closed-form solution of absolute orientation using unit quaternions, Journal of the Optical Society of America A, 4(4), 629-642.

4) Larsen, S., and Grieger, J., 1998, Elastic modeling initiative: Part III, 3-D computational modeling, 68th Annual International Meeting, SEG, Expanded Abstracts, 18031806.

5) Beyreuther, M., Barsch, R., Krischer, L., Megies, T., Behr, Y. and Wassermann, J., 2010, Obspy: A python toolbox for seismology, Seismological Research Letters, 81, 530-533. 